\title{
Journal of Nanoparticle Research: looking forward
}

\author{
Mihail C. Roco • Nicola Pinna
}

Published online: 4 March 2014

(C) Springer Science+Business Media Dordrecht 2014

Since 1999, the mission of the Journal of Nanoparticle Research has been to disseminate research on the physical, chemical and biological phenomena, and processes at work in nanoscale structures, i.e., where at least one dimension is in the range from atomic size and approximately $100 \mathrm{~nm}$. The focus of contributions has been toward improved or novel properties that are a direct result of their small size (Roco 1999). In the meantime, the field has evolved rapidly, and researchers are now ready to face new challenges. Our goal for the Journal in the years to come is to continue to support, nurture, and stimulate further research in the field as it moves in exciting new directions.

Over the years, the Journal's editorial policy has evolved, and the editorial board has become larger and more multidisciplinary. Here, we want to focus on some of the most important changes that have been implemented recently which directly affect authors and reviewers.

The Journal has published on an impressive range of synthetic procedures from the solid state, liquid, gas, and plasma phases, resulting in a large variety of

M. C. Roco ( $₫)$

National Science Foundation, 4201 Wilson Blvd., Arlington, VA, USA

e-mail: jnr.mroco@gmail.com; mroco@nsf.gov

\section{N. Pinna}

Institut für Chemie, Humboldt-Universität zu Berlin, Brook-Taylor-Str. 2, 12489 Berlin, Germany

e-mail: nicola.pinna@hu-berlin.de inorganic, organic and hybrid nanoscale, and nanostructured materials. The Journal is well established as a key reference source for synthesis and growth.

We are pleased to see that there is a large community of authors and readers interested in the Journal. Scholars in nearly 8,000 research institutions around the world have access to it. The number of submissions (from over 75 countries) has increased significantly every year, with an impressive 2149 submissions in 2013 (Table 1). Recent surveys indicate that the level of satisfaction among the Journal authors is rising, which we are delighted to see.

While nanomaterials syntheses and characterization continue to be important research topics, the scope of nanoparticle research goes well beyond this aspect. The Journal wishes to capture its full breadth and to promote the study of unprecedented physicochemical properties, fundamental transport phenomena, new interdisciplinary approaches toward assembling methods, manufacturing techniques, and applications in several areas of technology and healthcare.

To fulfill this aim, the Journal is more selective in the assessment of papers focused on syntheses and characterization of nanoparticles and nanostructures, maintaining the focus on highly original contributions. An increased emphasis will be on new areas of research that have emerged such as assembling into nanosystems, nanomanufacturing, nanophotonics and plasmonics, nanoenergy, nanofluidics, metamaterials, carbon nanotechnology, nanoecology, safety and 
Table 1 Some Journal performance indicators between 2009 and 2013

\begin{tabular}{lccccc}
\hline Indicators/year & 2009 & 2010 & 2011 & 2012 & 2013 \\
\hline $\begin{array}{l}\text { Number of } \\
\text { submissions }\end{array}$ & 777 & 1,215 & 1,474 & 1,782 & 2,149 \\
$\begin{array}{l}\text { Number of articles } \\
\text { published }\end{array}$ & 210 & 305 & 686 & 657 & 865 \\
$\begin{array}{c}\text { Acceptance rate } \\
\begin{array}{c}\text { Total number of } \\
\text { citations }\end{array}\end{array}$ & $37 \%$ & $35 \%$ & $36 \%$ & $38 \%$ & $38 \%$ \\
\hline
\end{tabular}

a Number of citations in the corresponding year of all articles published in JNR since 1999

b Source Web of Science, data collected January 31, 2014

nanotoxicity studies (with corresponding in vitro and in vivo testing methods), various branches of nanomedicine, opto-genetics, synthetic biology, and branches of sustainability. The Journal encourages both original papers and perspectives and reviews in these areas.

The continued growth in submission numbers is a challenge for both the editors and our reviewers. In the interest of keeping the standards of our reviewing process high, we have implemented some important editorial changes.

As of a year ago, authors are requested to submit a brief "scientific justification paragraph" to explain why they think their manuscript meets the scope and criteria set by the journal. Along with the manuscript, this text is an important element, required in order to consider the submission complete and suitable for peer review. This gives our authors the chance to tell more about the significance of the submitted work, beyond the highly specialized contents of the manuscript.

In order to focus the peer-review efforts on those manuscripts that match the scope and quality criteria of the Journal, it is necessary to conduct rigorous editorial screening at an early stage, before the referee's evaluation. Rejection decisions based on the editors' assessment have the advantage of saving precious time for authors, referees, and editors alike. This preliminary assessment ensures a fair evaluation of the manuscript by editors who are themselves scientists and experts in the field of the manuscript. (The editorial team of the Journal is composed of acknowledged researchers whose expertise spans the entire spectrum of sub-fields of nanoparticle science and technology.) We sincerely hope that our efforts to improve the Journal quality, reduce the reviewers' workload and shorten the overall processing time will be welcomed by the authors.

\section{Reference}

Roco MC (1999) Nanoparticles and nanotechnology research. J Nanopart Res 1:1-6 